\title{
NEFAs Influence the NF-kB Inflammatory and Insulin Signaling Pathways Through TLR4 in Bovine Hepatocytes In Vitro
}

Qinghua Deng ( $\sim$ dqh19850912@126.com )

Inner Mongolia University for Nationalities https://orcid.org/0000-0003-3625-112X

Liyin Du

Inner Mongolia University for Nationalities

Yuming Zhang

Inner Mongolia University for Nationalities

Guowen Liu

Jilin University

Research Article

Keywords: NEFAs, insulin resistance, NF-KB signaling pathway, lipid metabolism, bovine hepatocytes

Posted Date: June 2nd, 2021

DOI: https://doi.org/10.21203/rs.3.rs-553323/v1

License: (a) (i) This work is licensed under a Creative Commons Attribution 4.0 International License. Read Full License 


\section{Abstract}

Dairy cows are often in a state of negative energy balance (NEB), because of decreased dry matter intake and increased energy requirements, initiating lipid mobilization and leading to high serum $\beta$ hydroxybutyrate and nonesterified fatty acid (NEFA) levels, which can induce ketosis and fatty liver in dairy cows. We all have known that inflammation and insulin resistance are also common diseases in perinatal period of dairy cows. So what is the relationship between negative energy balance, insulin resistance and inflammation in cow? In order to study the role of NEFAs in the NF-KB inflammatory and insulin signaling pathways through TLR4, we cultured bovine primary hepatocytes and added different concentrations of NEFAs to test the mRNA and protein levels of inflammatory and insulin signaling pathways. Our experiments indicated that NEFAs could activate the NF-KB inflammatory signaling pathway and influence insulin resistance through TLR4. However, an inhibitor of TLR4 alleviated the inhibitory effects of NEFAs on the insulin pathway. In conclusion, all of these results indicate that highdose NEFAs can activate the TLR4/NF-KB inflammatory signaling pathway, and reduce the sensitivity of the insulin pathway through the TLR4/PI3K/AKT metabolic axis.

\section{Introdution}

Ketosis and fatty liver, common nutritional and metabolic diseases in perinatal cows, are characterized by negative energy balance (NEB) often accompanied by insulin resistance and inflammation [1]. Under NEB conditions, dairy cows mobilize their body fat to produce a large amount of nonesterified fatty acids (NEFAs) into the blood. Incompletely oxidized NEFAs in the liver may become $\beta$-hydroxybutyric acid (BHBA), the most important factor in ketosis, as excessive NEFAs oxidation can cause ketosis. When fatty acid metabolism accelerates, excess acetyl coenzyme $A$ produced by $\beta$-oxidation is transported to hepatocytes for ketosis. Fatty acids that do not undergo $\beta$-oxidation are re-esterified back into triglycerides (TGs) and released into the bloodstream in the form of very low-density lipoproteins. Studies in obese or high-fat diet-fed mice have demonstrated that NEFAs are closely related to inflammation and insulin resistance and act as signaling molecules that can directly regulate lipid metabolism in hepatocytes [2].

Insulin is a regulator of fat production [3]. In early lactation, the skeletal muscle and adipose tissue may exhibit hypoinsulinemia and decreased insulin sensitivity, resulting in increased glucose, which can be used by the breast [4]. Free fatty acids (FFAs) are increased in obese individuals and play an important

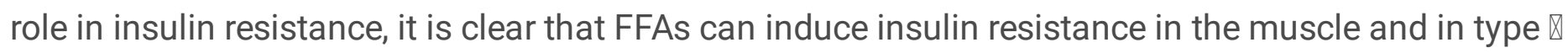
diabetes [5]. However, the mechanism by which FFAs induce NEB and liver insulin resistance in the perinatal period is not clear.

TLRs are a class of pathogen recognition receptors that activate the NF-KB signaling pathway and promote the transcription of pro-inflammatory factors [6]. Inflammatory factors can influence the transmission of insulin signaling pathway to downstream by different pathway to induce the phosphorylation of insulin receptor substrates at serine residues, which can reduce the physiological 
function of insulin and result in insulin resistance [7]. Recent studies indicate that TLR4 plays an important role in insulin resistance. In the tissue, skeletal muscle, pancreas and liver, insulin resistance is induced by the inflammatory response $[8,9]$. Furthermore, studies have indicated that NEFAs can activate TLR4 in vitro and in vivo [10]. However, no studies on the relationship between high-dose NEFAs, the TLR4/NF-KB inflammatory signaling pathway and liver insulin resistance, nor how NEFAs cause insulin resistance in bovine hepatocytes, have been carried out.

In this study, we added NEFAs to calf primary hepatocytes in vitro to detect the effects of NEFAs on inflammatory and insulin signaling pathways and illuminate whether NEFAs mediate cow hepatocyte inflammation and insulin resistance through TLR4.

\section{Results}

\section{NEFAs affected the NF-KB inflammatory pathway through the TLR4 protein}

We added TAK-242, and inhibitor of TLR4, and detected its effect on the inflammatory pathway. Hepatocytes were divided into four groups: NEFAs + TAK-242 group, TAK-242 group, NEFAs (2.4 mM) group and DMSO group. The TLR4 and p-P65 protein levels in the NEFAs group were higher than those in the DMSO group $(p<0.05)$; however, after addition of the inhibitor, namely, in the NEFAs + TAK-242 group, the protein level of TLR4 was significantly lower than that in the NEFAs group $(p<0.05)$. However, the level of P65 showed no obviously change $(p>0.05)$ (Fig. 3).

\section{Analysis of the effects of NEFAs on the NF-KB inflammatory pathways through TLR4 by cell immunofluorescence}

To further test whether NEFAs affects inflammatory pathways through TLR4, we used immunofluorescence to detect the effects of NEFAs and TAK-242 on the nuclear transfer of P65. The expression of P65 in the nucleus and cytoplasm was obviously higher in the NEFAs groups than that in the control group. The expression of P65 in the nucleus and cytoplasm was lower NEFAs + TAK-242 group than in the high-concentration group (Fig. 4).

\section{NEFAs affected inflammatory factors through TLR4 at the mRNA level}

We analyzed the effects of NEFAs on the inflammatory factors IL-6 and TNFa to see if they occurred through TLR4 at the mRNA level. The primer sequences specific for IL-6 and TNFa are listed in Table 1. The IL-6 mRNA level showed no obvious difference between low-concentration group and control group, but the medium- and high-concentration groups contained IL- 6 mRNA levels clearly higher than those in the control group $(p<0.05)$. However, the addition of TAK-242 obviously decreased the mRNA level of IL-6 
compared to that in the NEFAs group $(p<0.05)$. There was no difference in IL-6 mRNA level between the DMSO and control groups $(p>0.05)$ (Fig. 5A). The mRNA level of TNFa increased with increasing NEFA concentration $(p<0.05)$ and was highest in the high-concentration group $(p<0.05)$. Furthermore, the mRNA level of TNFa was lower in the TAK-242 + NEFAs group than in the high-concentration group and control group $(p<0.05)$, but there was no difference in TNFa mRNA levels between the TAK-242 and DMSO groups $(p>0.05)$ (Fig. 5B).

Table 1

Primers used for qRT-PCR

\begin{tabular}{|ll|}
\hline \multicolumn{2}{|l|}{ Primers used for PCR $\left(5^{\prime}\right.$ - $\left.3^{\prime}\right)$} \\
\hline B-actin & F: GCT AAC AGT CCG CCT AGA AGC A \\
\hline R: GTC ATC ACC ATC GGC AAT GAG \\
\hline F: AAC GAG TGG GTA AAG AAC GC \\
\hline R: CTG ACC AGA GGA GGG AAT GC \\
\hline F: CTG CCG GAC TAC CTG GAC TAT \\
\hline R6-Pase & F: AGC AAG TGG TTC CCG TTT C \\
\hline R: ACC CAG GCG AGG CAG TA \\
\hline F: AAG TAC CTT GAG GAG CAA GTG AA \\
\hline R: GGT GCG TTG TAT GGA TTG GA \\
\hline
\end{tabular}

\section{Effect of NEFAs on inflammatory cytokines in the cell supernatant}

To verify that NEFAs influence inflammatory pathways though TLR4, we detected the IL-6 and TNFa levels in the cell supernatant by ELISA. The IL- 6 and TNFa levels were obviously increased in the highconcentration group $(p<0.05)$ and lower in the TAK-242 + NEFAs group than in the high-concentration group $(p<0.05)$. However, there were no difference between the low- and medium-concentration groups and control group $(p>0.05)$ (Fig. 6).

\section{NEFAs effect the insulin pathway through TLR4 at the protein level}

In this experiment, we divided cells into 11 groups: the NEFAs group $(2.4 \mathrm{mM})$, TAK-242 group $(1 \mu \mathrm{M})$, NEFAs + TAK-242 group, DMSO group $(1 \mu \mathrm{M})$, INS group (100 nm), $0.6 \mathrm{mM}$ NEFAs + INS group, $1.2 \mathrm{mM}$ NEFAs + INS group, 2.4 mM NEFAs + INS group, TAK-242 + INS group, 2.4 mM NEFAs + TAK-242 + INS group, and DMSO + INS group. 
In the NEFAs group, the phosphorylation levels of GSK-3 $\beta$ and IRS1 were higher $(p<0.05)$, and the protein levels of p-AKT, p-IRS2, GLUT4 and PI3K were significantly lower than those in the DMSO group $(p<0.05)$. There were no significant difference in IRS1, IRS2 or AKT levels between the groups $(p>0.05)$. The protein level of GSK-3 $\beta$ in the NEFAs group was lower than that in the DMSO group $(p<0.05)$, but there was no difference between the other groups $(p>0.05)$. After the addition of the TLR4 inhibitor, the levels of phosphorylated IRS1 and GSK-3 $\beta$ in the NEFAs + TAK-242 group were lower than those in the NEFAs group; however, the protein levels of p-AKT, p-IRS2, GLUT4 and PI3K were significantly higher than those in the NEFAs group $(p<0.05)$. There was no difference in protein levels between the TAK-242 and DMSO groups $(p>0.05)$ except for those of $p-G S K-3 \beta$ and GLUT4 (Fig. 7).

We simultaneously added insulin to some cells as a control and found that the expression of p-IRS1 and IRS1 was no different between the groups with and without insulin $(p>0.05)$. The protein level of $p$-IRS2 increased as the NEFA concentration gradually increased but was lower in the high-concentration group than in the medium-concentration group and similar to the level in the low-concentration group. However, the expression of p-IRS2 in the NEFAs + TAK-242 group was lower than that in the 2.4 mM NEFAs group $(p<0.05)$. There was no difference in p-IRS2 levels between the DMSO and blank control groups, but it was higher in the TAK-242 group than in the blank control group. There was no difference in IRS2 level between the groups $(p>0.05)$. The PI3K protein level showed no obvious difference between the NEFAs groups ( $p>0.05$ ), but after the addition of TAK-242, it was higher than that in the 2.4 mM NEFAs group ( $p$ $<0.05)$. The expression levels of $\mathrm{p}-\mathrm{AKT}$ in the low- and medium-concentration groups were higher than that in the blank control group, but p-AKT expression in the high-concentration group was significantly lower than that in the medium- and low-concentration groups $(p<0.05)$. After the addition of TAK-242, the p-AKT level was obviously higher in the NEFAs + TAK-242 groups than in the high-concentration group ( $p$ $<0.05)$, and p-AKT levels in the TAK-242 and DMSO groups were higher than those in the blank control group $(p<0.05)$, but there was no difference in $\mathrm{p}-\mathrm{AKT}$ level between the TAK-242 and DMSO groups ( $p>$ $0.05)$. The protein level of AKT did not significantly differ between the NEFAs groups $(p>0.05)$ and was higher in the TAK-242 group than in the blank control group $(p<0.05)$. The protein level of $p$-GSK-3 $\beta$ increased as the NEFA concentration increased and was highest in the medium-concentration group but was lower in the high-concentration group than in the medium-concentration group $(p<0.05)$ and change in a dose-dependent manner. There was no significant difference in p-GSK-3 $\beta$ protein level between the other groups $(p>0.05)$. With increasing NEFA concentration, the protein level of $p-G S K-3 \beta$ increased and peaked in the medium-concentration group but was reduced in the high-concentration group, and this change was dose dependent. After the addition of an inhibitor, there was no significant difference in $p$ GSK-3 $\beta$ protein level between the groups $(p>0.05)$. The protein levels of GSK-3 $\beta$ in the low- and mediumconcentration groups were not significant different from that in the blank control group $(p>0.05)$, and the protein level in the high-concentration group was lower than that in the blank control group and NEFAs + TAK-242 group $(p<0.05)$. The protein expression of GLUT4 decreased with increasing NEFA concentration. After the addition of an inhibitor, GLUT4 protein expression was higher in the NEFAs + TAK242 group than in the high-concentration group $(p<0.05)$, and that in the TAK-242 and DMSO group did not significantly differ from that in the blank control group $(p>0.05)$ (Fig. 8). 


\section{NEFAs affect the expression of PEPCK and G6-Pase through TLR4 at the mRNA level}

PEPCK and G6-Pase are key enzymes in the gluconeogenesis process. The mRNA levels of PEPCK in the high- and medium-concentration groups were higher than that in the blank control group $(p<0.05)$, but there was no difference in PEPCK mRNA level between the low-concentration group and blank control group $(p>0.05)$. PEPCK mRNA levels in the NEFAs + TAK-242 group were lower than those in the blank control group, and there were no difference in PEPCK mRNA level between the TAK-242, DMSO and blank control groups $(p>0.05)$ (Fig. 9A). The mRNA level of G6-Pase increased with increasing NEFA concentration and was highest in the high-concentration group $(p<0.05)$. The expression level in the NEFAs + TAK-242 group was lower than that in the high-concentration group $(p<0.05)$, and there were no difference in expression between the other groups $(p>0.05)$ (Fig. 9B). After the addition of insulin, the mRNA level of PEPCK increased with increasing NEFA concentration, and PEPCK mRNA levels in the NEFAs + TAK-242 group were lower than those in the high-concentration group $(p<0.05)$, which was in accordance with the levels in the group without insulin (Fig. 10A). The mRNA level of G6-Pase reduced with increasing NEFA concentration; G6-Pase mRNA levels in the NEFAs + TAK-242 group were lower than those in the high-concentration group $(p<0.05)$, and there were no difference in G6-Pase level between TAK-242, DMSO and blank control groups ( $p>0.05)$ (Fig. 9B).

\section{Discussion}

Ketosis is one of the most common metabolic diseases in perinatal cows [11]. Furthermore, ketosisrelated diseases, including fatty liver, abomasum displacement, infectious diseases and other productive diseases, have long plagued agricultural development $[12,13]$. Perinatal cows are physically characterized by NEB [14]. The initial stage of NEB in cows is characterized by low glucose and high NEFA levels [15]. Cows under NEB conditions mobilize a large amount of fat to provide more ATP to oxidize NEFAs in the cow liver [16]. However, high NEFA concentrations in the blood of perinatal cows trigger some NEB-related metabolic disorders and diseases, such as liver lipid accumulation, ketosis, abomasum displacement and retained fetal membranes [17]. Clinical data show that fatty liver in cows often manifests as insulin resistance. Cows with type I ketosis often exhibit low insulin levels, and those with type II often exhibit high insulin levels with decreased insulin sensitivity. After recovery, the effects of insulin cannot correct NEB-mediated liver lipid metabolic disorder [18]. Therefore, specific liver regulation of the insulin signaling pathway is crucial to explaining the pathogenesis of cow ketosis and fatty liver. Postpartum cows often exhibit insulin resistance mainly caused by inadequate energy intake. However, the intake of too much energy may lead to obesity and insulin resistance, which are also closely related. TLRs are pattern recognition receptors that can affect microbial pathogens by promoting inflammatory signaling pathways such as the NF-KB pathway in the innate immune system [19]. The NF-kB pathway can promote the synthesis of inflammatory cytokines and chemokines [20]. Obesity is one of the most important factors that affects insulin resistance. Insulin resistance caused by obesity is associated with cytokines secreted by fat cells such as adiponectin and resistin and inflammatory factors such as TNF-a 
and IL-6 [21]. Obesity is characterized by long-term inflammatory pathway activation, and inflammatory diseases are associated with insulin resistance in obesity. However, the mechanism of this inflammatory pathway activation is not clear in obese patients. The levels of blood FFAs in obese patients tend to be increased, and a variety of hypotheses regarding insulin resistance caused by FFAs have now been proposed. High NEFA and BHBA levels are clinicopathological characteristics of NEB; however, it is unclear whether insulin resistance in perinatal cows with NEB is associated with high blood NEFA levels.

\section{NEFAs affect the NF-KB inflammatory signaling pathway through TLR4}

Studies in humans and mice have shown a very close connection between inflammation and insulin resistance [22-24]. Rats in which TLR4 was specifically knocked out fed a high-fat diet exhibit fatty liver, but this high-fat diet can obviously increase insulin sensitivity, reducing fat accumulation and inflammation in the blood [25]. The metabolic characteristics of cows are different from those of humans and rats, and the relationship between insulin resistance and inflammation in bovines is not clear. In this study, we assumed that NEFAs can bind TLR4 on the cell membrane of hepatocytes and influence the NF$\mathrm{KB}$ and insulin signaling pathways.

We found that NEFAs obviously increased the initial calf hepatocyte TLR4 protein expression level, and with increasing concentrations of NEFAs, the P65 phosphorylation level also gradually increased (Fig. 1). Because the activation of NF-KB is mainly achieved through the phosphorylation of p65, NEFAs can activate the TLR4 and NF-KB pathways. After activation of the NF-kB pathway, a large number of inflammatory factors such as IL-6, IL-1 $\beta$, and TNF- $\alpha$ are produced. To detect whether NEFAs affect the NFKB inflammatory pathway by binding TLR4, we added a TLR4 inhibitor group and the corresponding control group (Fig. 3). Our experimental results showed that the protein expression of TLR4 and p-P65 in the group mixed with a TLR4 inhibitor and NEFAs was significantly lower than that in the NEFAs group, and nuclear expression of the nuclear transcription factor p65 decreased significantly after the addition of inhibitor (Fig. 4). Similarly, the mRNA expression and supernatant concentration of the proinflammatory factors IL- 6 and TNF-a, were significantly increased in the NEFAs group but decreased after the addition of TLR4 inhibitors (Fig. 5,6). This indicates that the inhibitor of TLR4 could inhibit not only TLR4 but also P65, a key factor in the inflammatory pathway of NF-KB, and can also inhibit the expression and secretion of proinflammatory factors. Overall, these findings indicate that NEFAs can activate the NF-KB pathway through TLR4, promote the release of proinflammatory factors such as IL-6 and TNF-a, and then promote the inflammatory response.

\section{NEFAs affect insulin pathway through TLR4}

In patients with diabetes, metabolically active cells cannot use glucose, so they heavily accumulate in the blood [26]. Glucose is mainly used in targeted tissues through two pathways-the PI3K and AMPK pathways [27]. The PI3K/AKT pathway promotes the expression of GLUT4 in muscle cells and adipocytes and plays an important role in glucose metabolism [28]. AMPK is activated mainly through drug 
stimulation, such as stimulation with metformin, or through exercise, effectively promoting glucose utilization and insulin sensitivity [29]. Therefore, the PI3K/AKT and AMPK pathways may be potential targets for regulating insulin resistance to glucose metabolism in type 2 diabetes and obesity. In this study, we studied the PI3K/AKT pathway. IRS1 is a key regulatory factor downstream of the insulin receptor in insulin signaling. IRS1-deficient mice showed normal glucose tolerance and insulin resistance [30]. Nakamura A (2012) et al indicated that although severe hyperinsulinemia occurred in IRS1-/-rats fed a high-fat diet, there was no liver lipid degeneration [31]. The activation of PI3K in the PI3K pathway depends on the binding of two SH2 regions to the tyrosine-phosphorylated IRS protein [32]. Knockout of the PI3K regulatory subunits $p 85 a$ and $p 85 \beta$ increased insulin sensitivity $[33,34]$. Previous studies have shown that liver-specific knockout of PRSS8 reduced the phosphorylation of AKT in the liver, resulting in insulin resistance [35]. GSK-3 $\beta$ is a key enzyme that regulates insulin signaling through glycogen

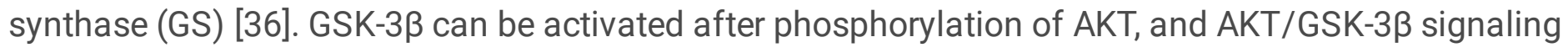
plays an important role in insulin resistance [37].

In this study, we first examined whether NEFAs could affect the insulin pathway in primary calf hepatocytes. High concentrations of NEFAs increased the protein expression of p-IRS1 (Ser307), p-IRS2, IRS2 and GLUT4 but decreased the expression of PI3K, p-AKT and p-GSK (Fig. 2). Therefore, we determined that high concentrations of NEFAs can induce insulin resistance in primary calf hepatocytes. To determine whether NEFAs affect the insulin pathway through TLR4, we detected changes in the expression of key proteins in the insulin pathway after adding TLR4 inhibitors and prepared a non-insulin group as a control. After the addition of insulin, the expression levels of key proteins in the insulin pathway were greater than those in the non-insulin group; for example, the decrease in PI3K and p-AKT was more pronounced than that in the non-insulin group (Fig. 7, 8). In general, the addition of TLR4 inhibitors affected the insulin pathway, indicating that NEFAs can affect the insulin pathway by binding TLR4. PEPCK and G6-Pase are two key enzymes involved in gluconeogenesis. Previous studies have shown that the activation of AKT in mouse liver can induce insulin-mediated gluconeogenesis, accompanied by downregulation of PEPCK and G6-Pase expression [38]. To further verify the effect of TLR4 inhibitors on genes downstream of the insulin pathway, we detected the mRNA expression levels of PEPCK and G6-Pase in hepatocytes. In the absence of insulin, the mRNA expression levels of PEPCK and G6-Pase were increased in the NEFAs group and decreased in the inhibitor mixed group, but after the addition of insulin, G6-Pase showed the opposite change (Figs. 9, 10). Because PEPCK and G6-Pase are downstream of the insulin pathway, their sensitivity to insulin is not particularly accurate, so this finding is not enough to explain its effect on the insulin pathway.

Consequently, these results indicate that NEFAs can regulate the insulin pathway through the TLR4/PI3K/AKT metabolic axis.

\section{Declarations}

\section{Author contribution}


Qinghua Deng囚contributed to the conception of the study and wrote the manuscript.

Liyin Du: performed the data analyses.

Yuming Zhang: performed the experiment.

Guowen Liu: helped perform the analysis with constructive discussions.

\section{Acknowledgments}

This work was supported by the National Natural Science Foundation of China (Beijing, China; grant no. 31602121 and 31760752), Young scientific and technological talents in Inner Mongolia (Inner Mongolia, china, grant no. NJYT-20-B30), Inner Mongolia Beef Diseases Prevention and Control Engineering Technology Research Center (grant no. MDK2019023), Dr start-up fund in Inner Mongolia University for Nationalities (BS476).

\section{Conflicts of interest}

The authors declare no conflict of interest.

Availability of data and material The datasets generated during and/or analyzed dur-ing the current study are available from the corresponding author on reasonable request.

Funding Not applicable.

Code availability Not applicable.

Ethics approval The study protocol was approved by the Ethics Committee on the Use and Care of Animals at the Inner Mongolia University for Nationalities (Tongliao, China).

Consent to participate Not applicable.

Consent for publication Not applicable.

\section{References}

1. Deng Q, Li X, Fu S, Yin L, Zhang Y, Wang T, Wang J, Liu L, Yuan X, Sun G, Wang Z, Liu G, Li X (2014) SREBP-1c gene silencing can decrease lipid deposits in bovine hepatocytes cultured in vitro. Cell Physiol Biochem 33:1568-1578. doi:10.1159/000358720

2. Airaksinen K, Jokkala J, Ahonen I, Auriola S, Kolehmainen M, Hanhineva K, Tiihonen K (2018) HighFat Diet, Betaine, and Polydextrose Induce Changes in Adipose Tissue Inflammation and Metabolism in C57BL/6J Mice. Mol Nutr Food Res 62:e1800455. doi:10.1002/mnfr.201800455

3. Huang Z, Lu X, Huang L, Zhang C, Veldhuis JD, Cowley MA, Chen C (2021) Stimulation of endogenous pulsatile growth hormone secretion by activation of growth hormone secretagogue 
receptor reduces the fat accumulation and improves the insulin sensitivity in obese mice. FASEB $J$ 35:e21269. doi:10.1096/fj.202001924RR

4. Lu N, Zhang M, Lu L, Liu YZ, Liu XD, Zhang HH (2020) Insulin-Induced Gene 2 Expression Is Associated with Breast Cancer Metastasis. Am J Pathol. doi:10.1016/j.ajpath.2020.11.013

5. Rachek LI (2014) Free fatty acids and skeletal muscle insulin resistance. Prog Mol Biol Transl Sci 121:267-292. doi:10.1016/B978-0-12-800101-1.00008-9

6. Li X, Huang R, Liu K, Li M, Luo H, Cui L, Huang L, Luo L (2020) Fucoxanthin attenuates LPS-induced acute lung injury via inhibition of the TLR4/MYD88 signaling axis. Aging (Albany NY) 12. doi: 10.18632/aging.202309

7. Dludla PV, Nkambule BB, Mazibuko-Mbeje SE, Nyambuya TM, Marcheggiani F, Cirilli I, Ziqubu K, Shabalala SC, Johnson R, Louw J, Damiani E, Tiano L (2020) N-Acetyl Cysteine Targets Hepatic Lipid Accumulation to Curb Oxidative Stress and Inflammation in NAFLD: A Comprehensive Analysis of the Literature. Antioxidants (Basel) 9. doi:10.3390/antiox9121283

8. Oliveira AG, Carvalho BM, Tobar N, Ropelle ER, Pauli JR, Bagarolli RA, Guadagnini D, Carvalheira JB, Saad MJ (2011) Physical exercise reduces circulating lipopolysaccharide and TLR4 activation and improves insulin signaling in tissues of DIO rats. Diabetes 60:784-796. doi:10.2337/db09-1907

9. Saberi M, Woods NB, de Luca C, Schenk S, Lu JC, Bandyopadhyay G, Verma IM, Olefsky JM (2009) Hematopoietic cell-specific deletion of toll-like receptor 4 ameliorates hepatic and adipose tissue insulin resistance in high-fat-fed mice. Cell Metab 10:419-429. doi:10.1016/j.cmet.2009.09.006

10. Tamer F, Ulug E, Akyol A, Nergiz-Unal R (2020) The potential efficacy of dietary fatty acids and fructose induced inflammation and oxidative stress on the insulin signaling and fat accumulation in mice. Food Chem Toxicol 135:110914. doi:10.1016/j.fct.2019.110914

11. Soares RAN, Vargas G, Muniz MMM, Soares MAM, Canovas A, Schenkel F, Squires EJ (2021) Differential gene expression in dairy cows under negative energy balance and ketosis: A systematic review and meta-analysis. J Dairy Sci 104:602-615. doi:10.3168/jds.2020-18883

12. Herdt TH (2000) Ruminant adaptation to negative energy balance. Influences on the etiology of ketosis and fatty liver. Vet Clin North Am Food Anim Pract 16:215-230, v

13. Oetzel GR (2004) Monitoring and testing dairy herds for metabolic disease. Veterinary Clinics Of North America-Food Animal Practice 20:651-674. DOI 10.1016/j.cvfa.2004.06.006 doi

14. Dann HM, Drackley JK (2005) Carnitine palmitoyltransferase I in liver of periparturient dairy cows: effects of prepartum intake, postpartum induction of ketosis, and periparturient disorders. J Dairy Sci 88:3851-3859. doi:10.3168/jds.S0022-0302(05)73070-8

15. Murondoti A, Jorritsma R, Beynen AC, Wensing T, Geelen MJ (2004) Unrestricted feed intake during the dry period impairs the postpartum oxidation and synthesis of fatty acids in the liver of dairy cows. J Dairy Sci 87:672-679. doi:10.3168/jds.S0022-0302(04)73210-5

16. Greenfield RB, Cecava MJ, Donkin SS (2000) Changes in mRNA expression for gluconeogenic enzymes in liver of dairy cattle during the transition to lactation. J Dairy Sci 83:1228-1236. doi:10.3168/jds.S0022-0302(00)74989-7 
17. Xu C, Wang Z, Liu GW, Li XB, Xie GH, Xia C, Zhang HY (2008) Metabolic characteristic of the liver of dairy cows during ketosis based on comparative proteomics. Asian-Australasian Journal Of Animal Sciences 21:1003-1010

18. Li X, Li G, Du X, Sun X, Peng Z, Zhao C, Xu Q, Abdelatty AM, Mohamed FF, Wang Z, Liu G (2020) Increased autophagy mediates the adaptive mechanism of the mammary gland in dairy cows with hyperketonemia. J Dairy Sci 103:2545-2555. doi:10.3168/jds.2019-16910

19. Wang Z, Ni X, Zhang L, Sun L, Zhu X, Zhou Q, Yang Z, Yuan H (2020) Toll-Like Receptor 4 and Inflammatory Micro-Environment of Pancreatic Islets in Type-2 Diabetes Mellitus: A Therapeutic Perspective. Diabetes Metab Syndr Obes 13:4261-4272. doi:10.2147/DMSO.S279104

20. Bertelsen A, Elborn SJ, Schock BC (2020) Toll like Receptor signalling by Prevotella histicola activates alternative NF-kappaB signalling in Cystic Fibrosis bronchial epithelial cells compared to $P$. aeruginosa. PLoS One 15:e0235803. doi:10.1371/journal.pone.0235803

21. Lima MM, Pareja JC, Alegre SM, Geloneze SR, Kahn SE, Astiarraga BD, Chaim EA, Baracat J, Geloneze B (2013) Visceral fat resection in humans: effect on insulin sensitivity, beta-cell function, adipokines, and inflammatory markers. Obesity (Silver Spring) 21:E182-E189. doi:10.1002/oby.20030

22. Al-Sofiani ME, Jammah A, Racz M, Khawaja RA, Hasanato R, El-Fawal HA, Mousa SA, Mason DL (2015) Effect of Vitamin D Supplementation on Glucose Control and Inflammatory Response in Type II Diabetes: A Double Blind, Randomized Clinical Trial. Int J Endocrinol Metab 13:e22604. doi:10.5812/ijem.22604

23. Kim MJ, Rangasamy S, Shim Y, Song JM (2015) Cell lysis-free quantum dot multicolor cellular imaging-based mechanism study for TNF-alpha-induced insulin resistance. J Nanobiotechnology 13:4. doi:10.1186/s12951-015-0064-x

24. Le NH, Kim CS, Park T, Park JH, Sung MK, Lee DG, Hong SM, Choe SY, Goto T, Kawada T, Yu R (2014) Quercetin protects against obesity-induced skeletal muscle inflammation and atrophy. Mediators Inflamm 2014:834294. doi:10.1155/2014/834294

25. Jia L, Vianna CR, Fukuda M, Berglund ED, Liu C, Tao C, Sun K, Liu T, Harper MJ, Lee CE, Lee S, Scherer PE, Elmquist JK (2014) Hepatocyte Toll-like receptor 4 regulates obesity-induced inflammation and insulin resistance. Nat Commun 5:3878. doi:10.1038/ncomms4878

26. Chehade JM, Gladysz M, Mooradian AD (2013) Dyslipidemia in type 2 diabetes: prevalence, pathophysiology, and management. Drugs 73:327-339. doi:10.1007/s40265-013-0023-5

27. Cordero-Herrera I, Martin MA, Bravo L, Goya L, Ramos S (2013) Cocoa flavonoids improve insulin signalling and modulate glucose production via AKT and AMPK in HepG2 cells. Mol Nutr Food Res 57:974-985. doi:10.1002/mnfr.201200500

28. Bryant NJ, Govers R, James DE (2002) Regulated transport of the glucose transporter GLUT4. Nat Rev Mol Cell Biol 3:267-277. doi:10.1038/nrm782

29. Coughlan KA, Valentine RJ, Ruderman NB, Saha AK (2014) AMPK activation: a therapeutic target for type 2 diabetes? Diabetes Metab Syndr Obes 7:241-253. doi:10.2147/DMSO.S43731 
30. Saltiel AR, Kahn CR (2001) Insulin signalling and the regulation of glucose and lipid metabolism. Nature 414:799-806. doi:10.1038/414799a

31. Nakamura A, Tajima K, Zolzaya K, Sato K, Inoue R, Yoneda M, Fujita K, Nozaki Y, Kubota KC, Haga H, Kubota N, Nagashima Y, Nakajima A, Maeda S, Kadowaki T, Terauchi Y (2012) Protection from nonalcoholic steatohepatitis and liver tumourigenesis in high fat-fed insulin receptor substrate-1knockout mice despite insulin resistance. Diabetologia 55:3382-3391. doi:10.1007/s00125-0122703-1

32. Shaw LM (2011) The insulin receptor substrate (IRS) proteins: at the intersection of metabolism and cancer. Cell Cycle 10:1750-1756

33. Terauchi Y, Tsuji Y, Satoh S, Minoura H, Murakami K, Okuno A, Inukai K, Asano T, Kaburagi Y, Ueki K, Nakajima H, Hanafusa T, Matsuzawa Y, Sekihara H, Yin Y, Barrett JC, Oda H, Ishikawa T, Akanuma Y, Komuro I, Suzuki M, Yamamura K, Kodama T, Suzuki H, Yamamura K, Kodama T, Suzuki H, Koyasu S, Aizawa S, Tobe K, Fukui Y, Yazaki Y, Kadowaki T (1999) Increased insulin sensitivity and hypoglycaemia in mice lacking the p85 alpha subunit of phosphoinositide 3-kinase. Nat Genet 21:230-235. doi:10.1038/6023

34. Ueki K, Yballe CM, Brachmann SM, Vicent D, Watt JM, Kahn CR, Cantley LC (2002) Increased insulin sensitivity in mice lacking p85beta subunit of phosphoinositide 3-kinase. Proc Natl Acad Sci U S A 99:419-424. doi:10.1073/pnas.012581799

35. Uchimura K, Hayata M, Mizumoto T, Miyasato Y, Kakizoe Y, Morinaga J, Onoue T, Yamazoe R, Ueda M, Adachi M, Miyoshi T, Shiraishi N, Ogawa W, Fukuda K, Kondo T, Matsumura T, Araki E, Tomita K, Kitamura K (2014) The serine protease prostasin regulates hepatic insulin sensitivity by modulating TLR4 signalling. Nat Commun 5:3428. doi:10.1038/ncomms4428

36. Rayasam GV, Tulasi VK, Sodhi R, Davis JA, Ray A (2009) Glycogen synthase kinase 3: more than a namesake. Br J Pharmacol 156:885-898. doi:10.1111/j.1476-5381.2008.00085.x

37. Lee J, Kim MS (2007) The role of GSK3 in glucose homeostasis and the development of insulin resistance. Diabetes Res Clin Pract 77(Suppl 1):S49-S57. doi:10.1016/j.diabres.2007.01.033

38. Brust KB, Corbell KA, Al-Nakkash L, Babu JR, Broderick TL (2014) Expression of gluconeogenic enzymes and 11beta-hydroxysteroid dehydrogenase type 1 in liver of diabetic mice after acute exercise. Diabetes Metab Syndr Obes 7:495-504. doi:10.2147/DMSO.S70767

\section{Figures}


NEFAs (mM) $0 \quad 0.6 \quad 1.2 \quad 2.4$

INS - - - - -

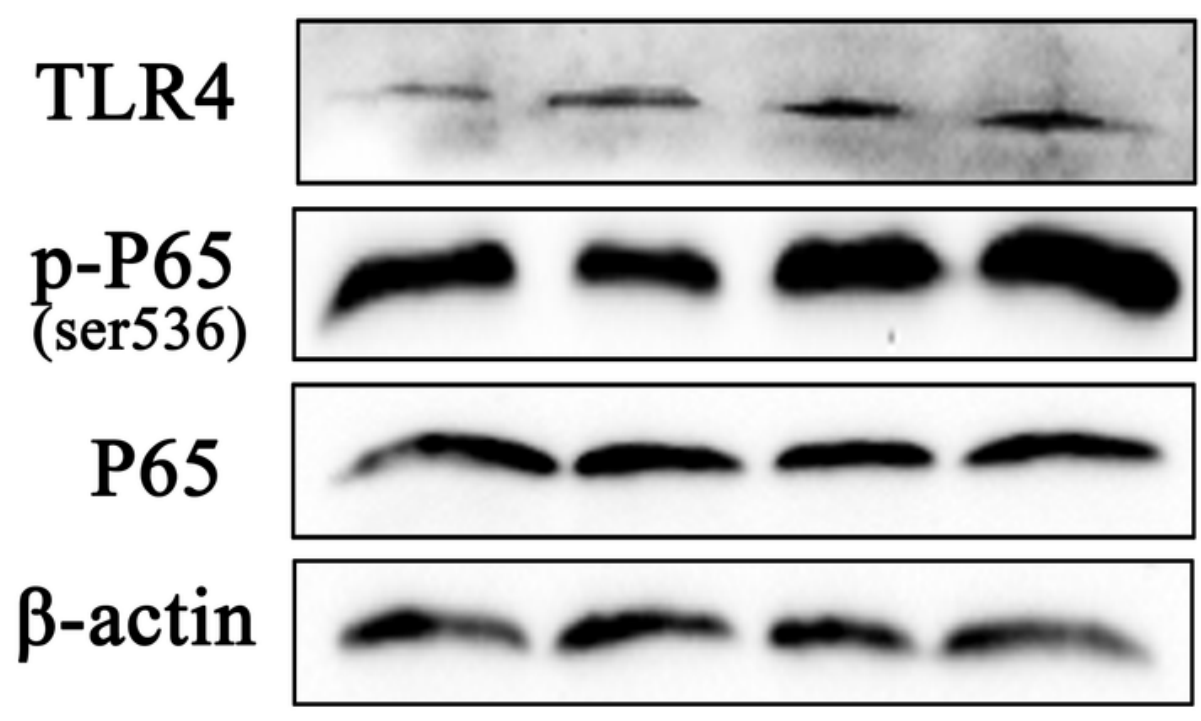

\section{Figure 1}

Effects of NEFAs on TLR4 and the inflammatory pathway. Protein expression levels after incubation with NEFAs were determined by western blotting. Hepatocytes were divided into four groups: the control group (0 mM NEFAs), low-concentration group (0.6 mM NEFAs), medium-concentration group (1.2 mM NEFAs) and high-concentration group (2.4 mM NEFAs). 
$\begin{array}{lllll}\text { NEFAs (mM) } & 0 & 0.6 & 1.2 & 2.4\end{array}$

INS

p-IRS1

(ser307)

IRS1

p-IRS2

(ser731)

IRS2

PI3K

p-AKT

AKT

p-GSK-3 $\beta$

(ser9)

GSK-3 $\beta$

GLUT4

$\beta$-actin
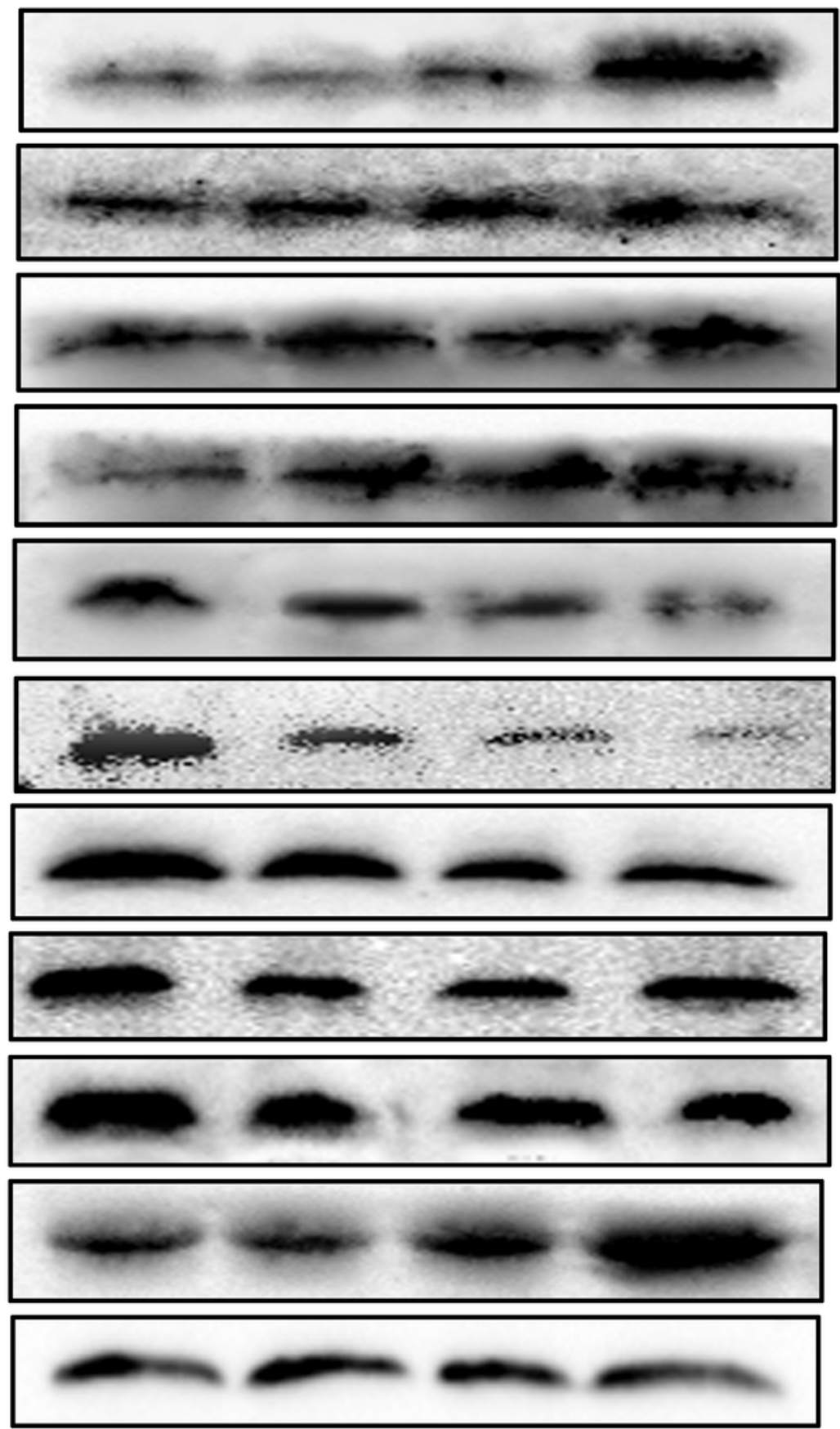

Figure 2

Effects of NEFAs on the expression of related proteins in the insulin signaling pathway. Protein expression levels after incubation with NEFAs were determined by western blotting. Hepatocytes were divided into four groups: the control group (0 mM NEFAs), low-concentration group (0.6 mM NEFAs), medium-concentration group (1.2 mM NEFAs) and high-concentration group (2.4 mM NEFAs). 


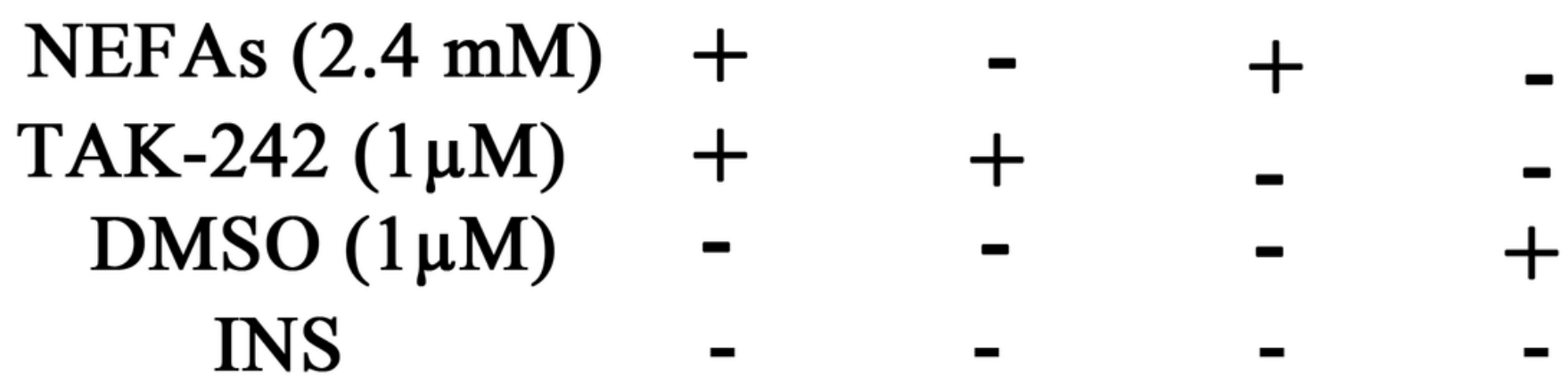

\section{TLR4 p-P65 (ser536) P65 $\beta$-actin}

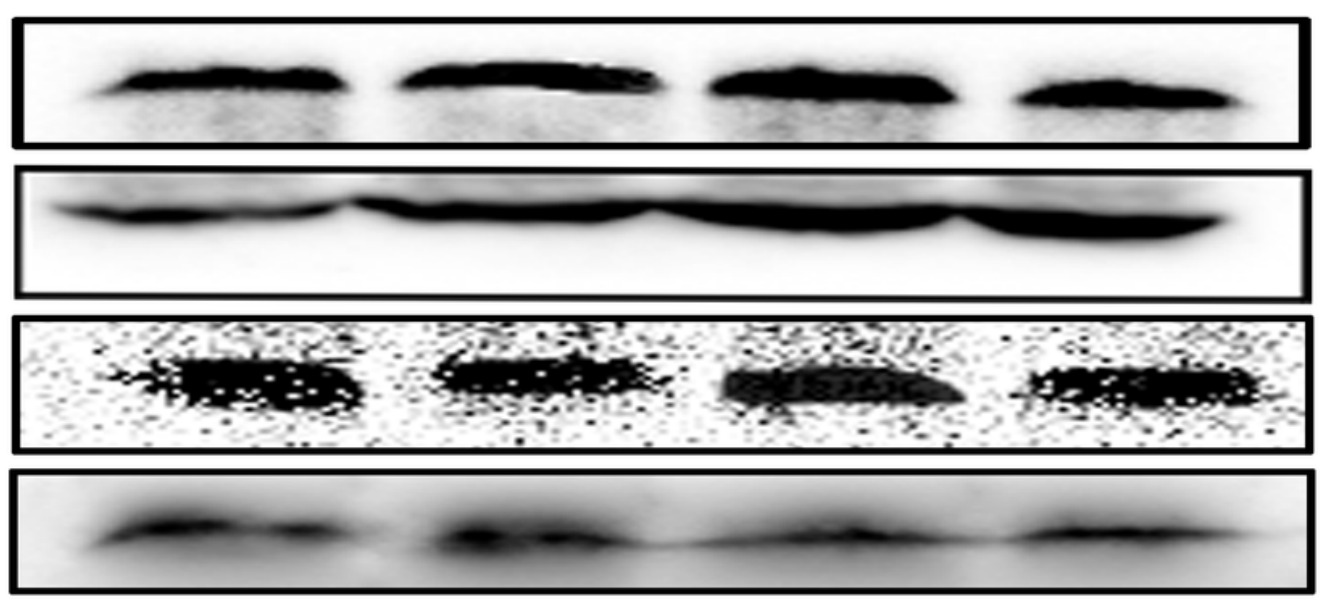

Figure 3

NEFAs affected the NF-KB inflammatory pathway through the TLR4 protein. Protein expression levels were determined by western blotting. Hepatocytes were divided into four groups: the NEFAs+TAK-242 group, TAK-242 group, NEFAs (2.4 mM) group and DMSO group. 


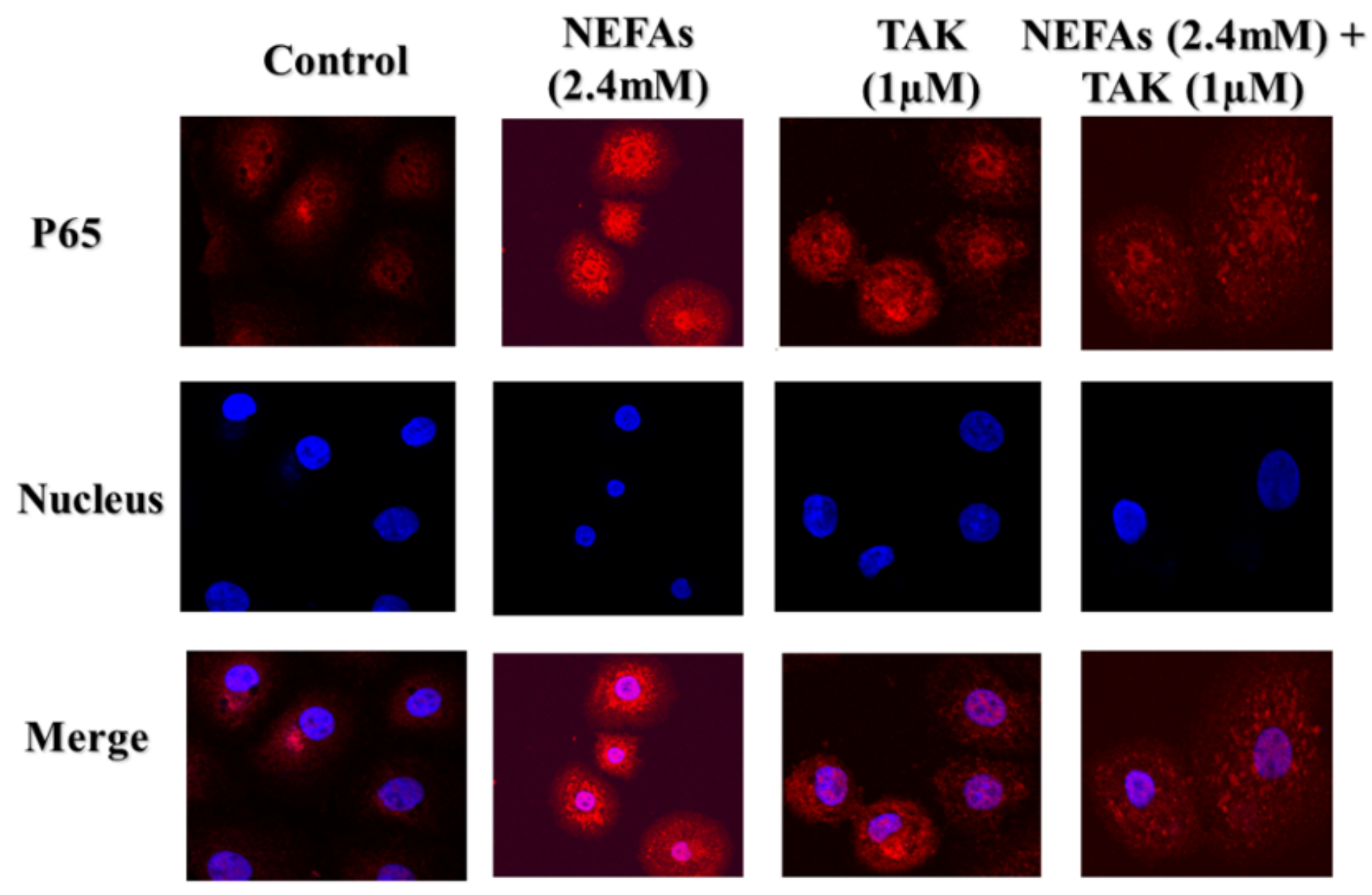

Figure 4

Results of immunofluorescence analysis of P65 in bovine hepatocytes (800x). Hepatocytes were divided into four groups: NEFAs+TAK-242 group, TAK-242 group, NEFAs (2.4 mM) group and DMSO group.

A

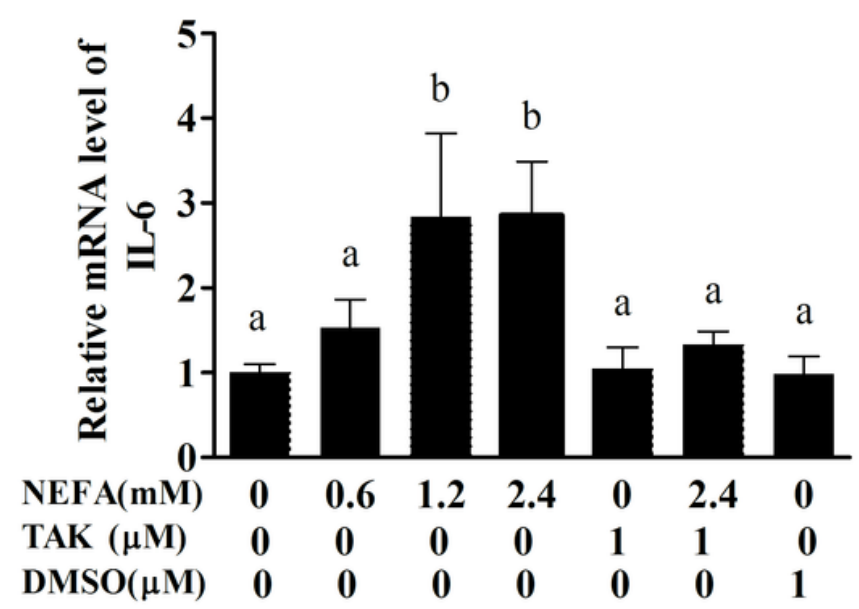

B

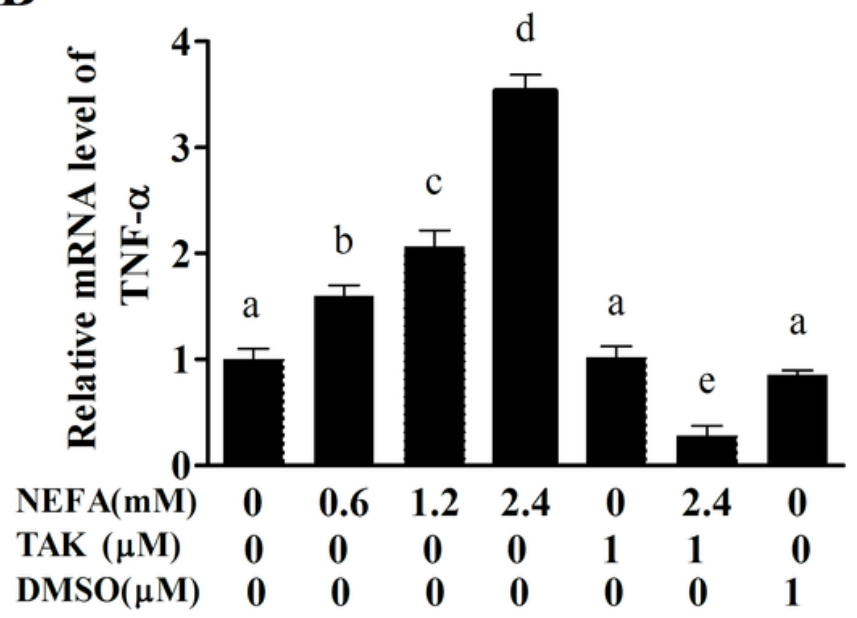

Figure 5 
Effects of NEFAs and TAK-242 on the mRNA expression levels of IL- 6 and TNFa Values are the means \pm SEs $(n=6)$, and different letters indicate a statistically significant difference; $p<0.05$.

A

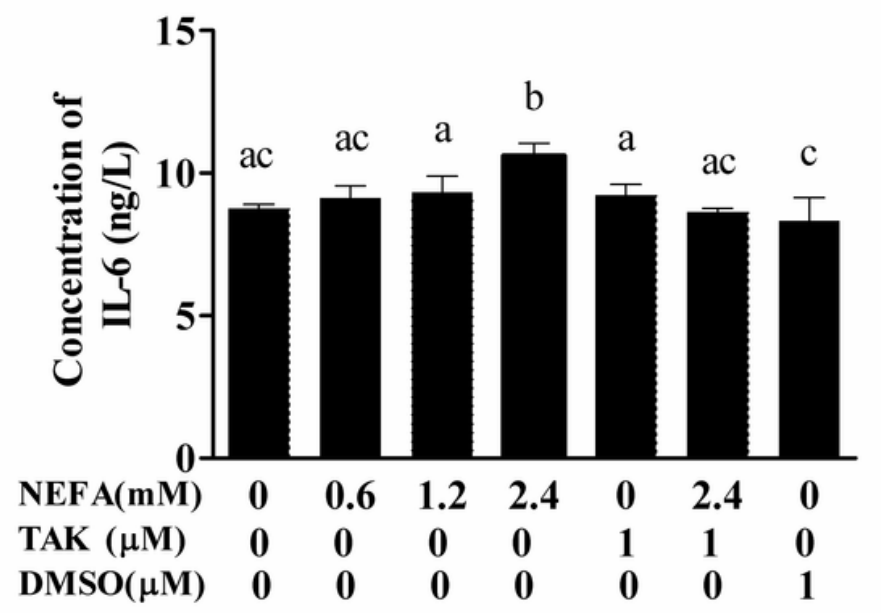

B

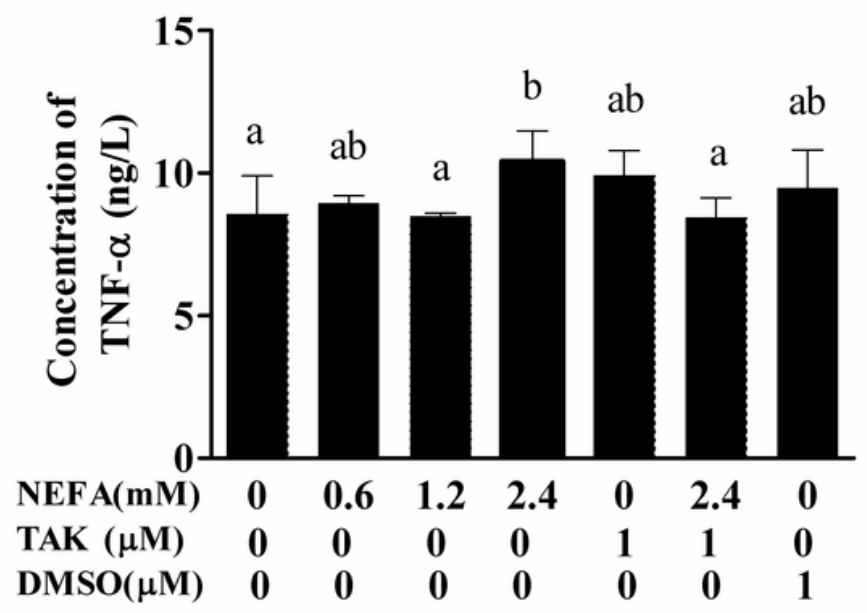

Figure 6

Effects of NEFAs on IL-6 and TNFa in the culture supernatant Values are the means \pm SEs $(n=6)$, and different letters indicate statistically significant differences; $p<0.05$. 


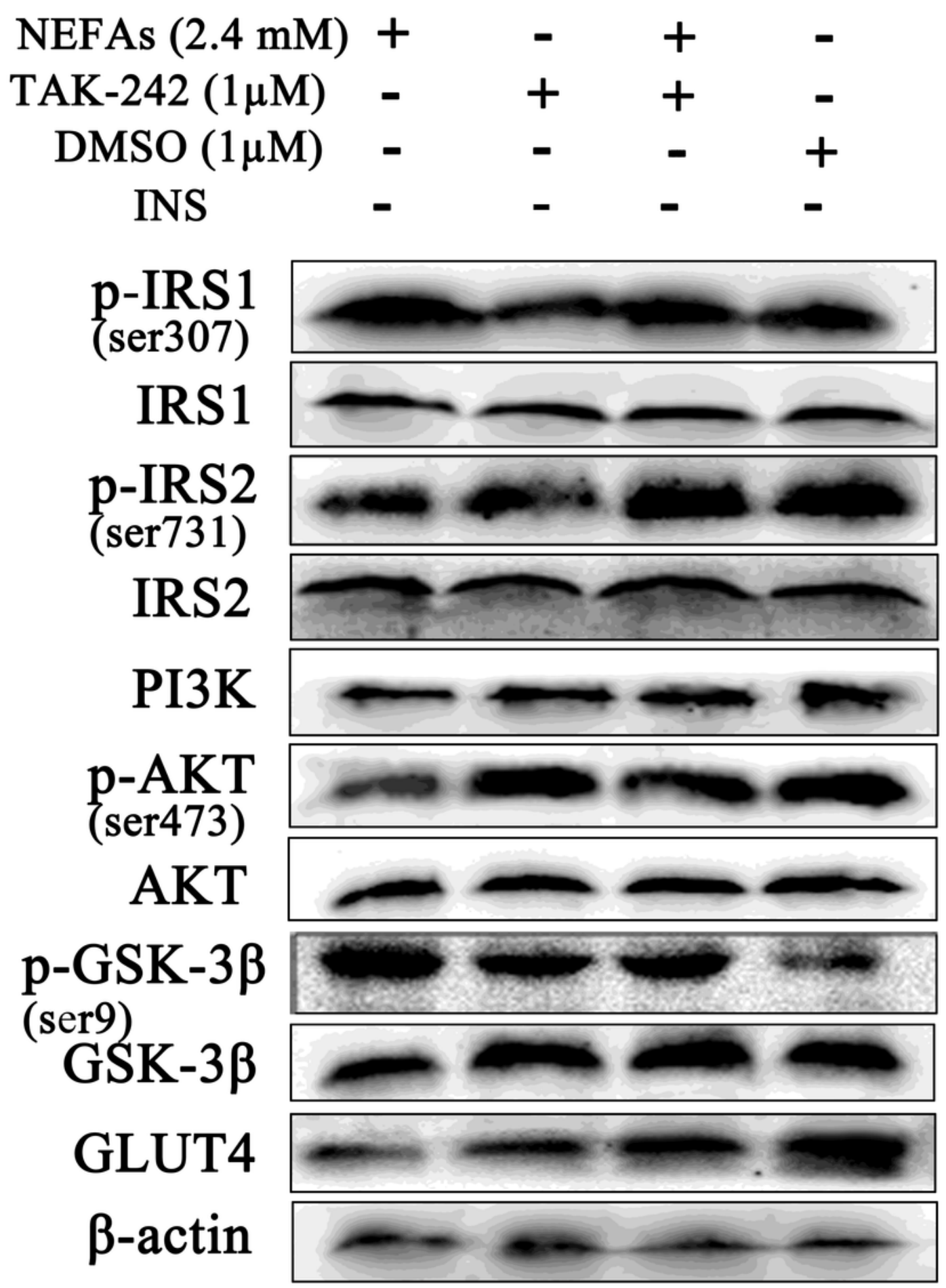

Figure 7

Effects of NEFAs and TAK-242 on key proteins in the insulin signaling pathway 
$\begin{array}{llllllll}\text { NEFAs }(m M) & 0 & 0.6 & 1.2 & 2.4 & - & 2.4 & -\end{array}$ $\begin{array}{rccccccc}\text { TAK-242 }(1 \mu \mathrm{M}) & - & - & - & - & + & + & - \\ \operatorname{DMSO}(1 \mu \mathrm{M}) & - & - & - & - & - & - & +\end{array}$ INS (100nM) +++++++ p-IRS1 (ser307)

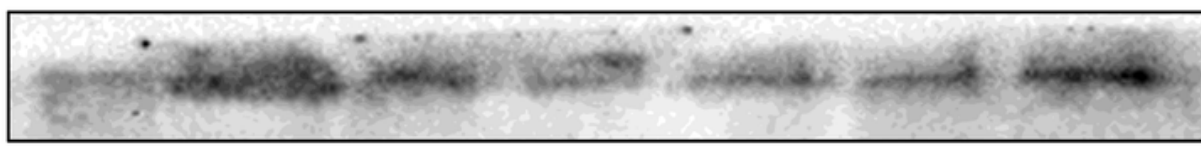

IRS1

p-IRS2 (ser731)

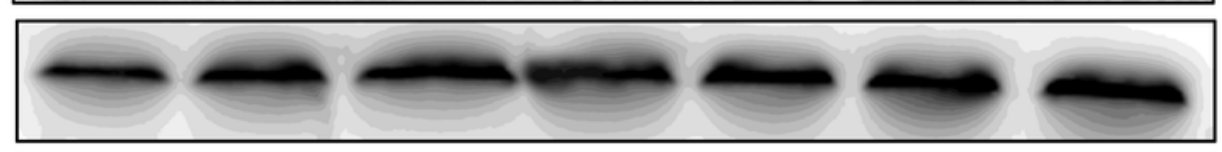

IRS2

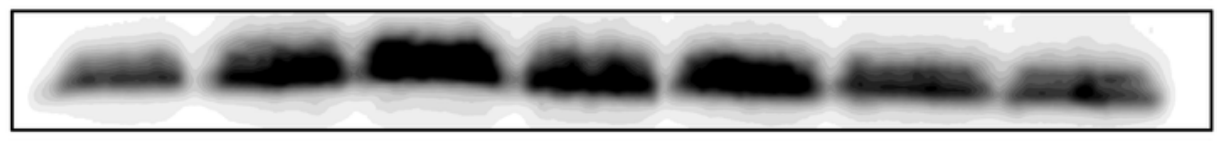

AKT
p-GSK-3 $\beta$
(ser9)
GSK-3 $\beta$

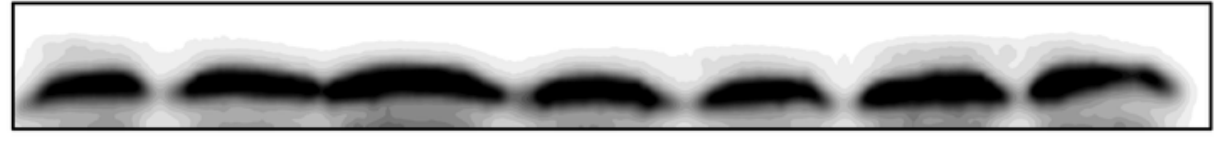
PI3K p-AKT
(ser473)

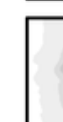

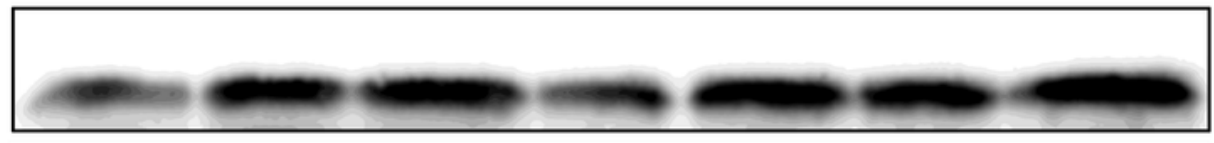

\section{GLUT4} $\beta$-actin
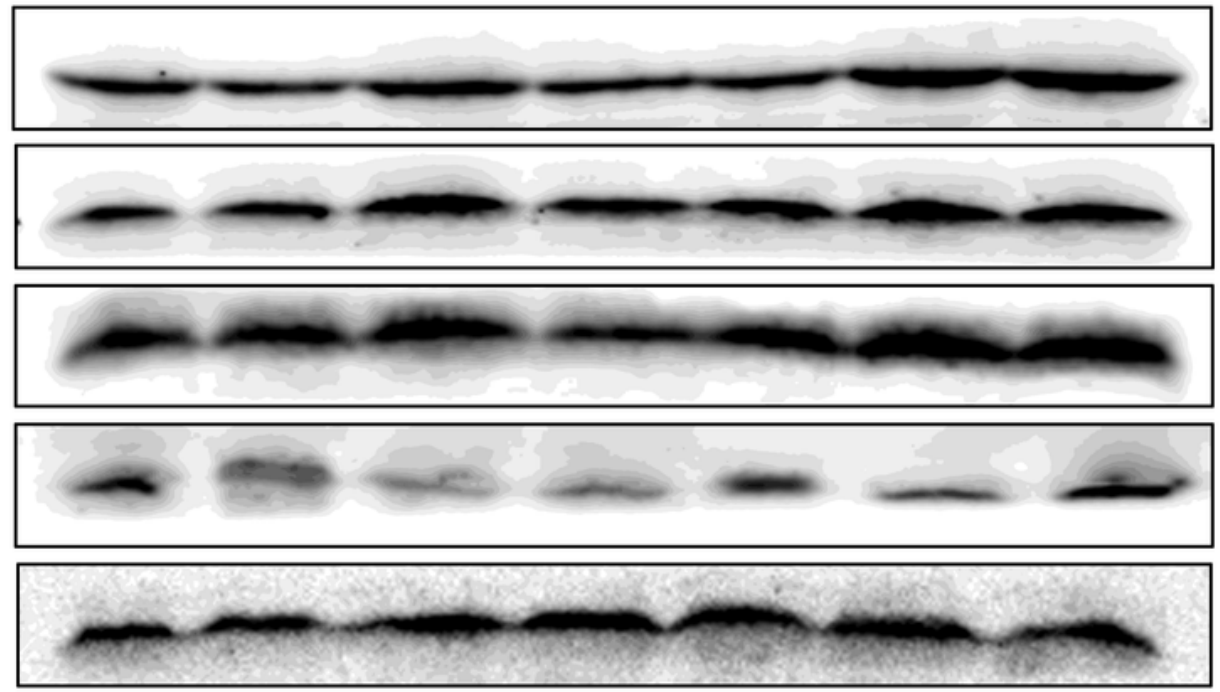

Figure 8

Effects of NEFAs, TAK-242 and INS on key proteins in the insulin signaling pathway 
A

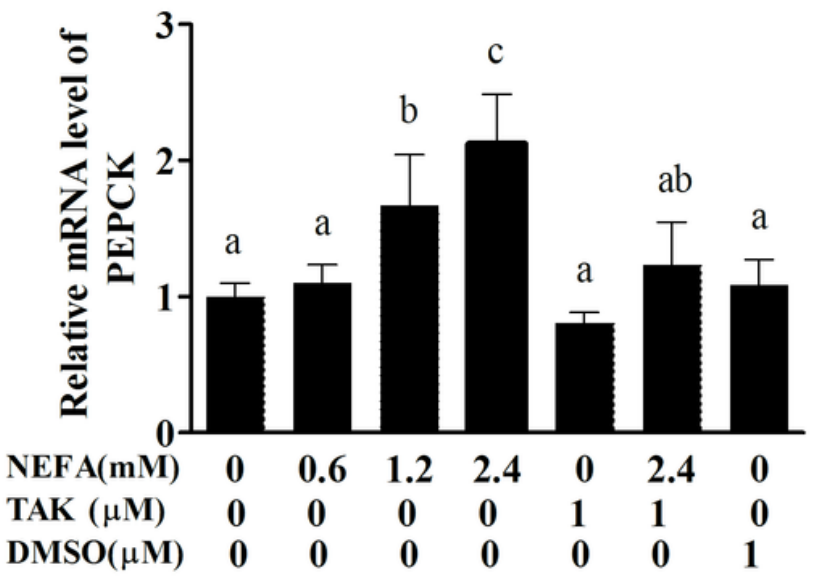

B

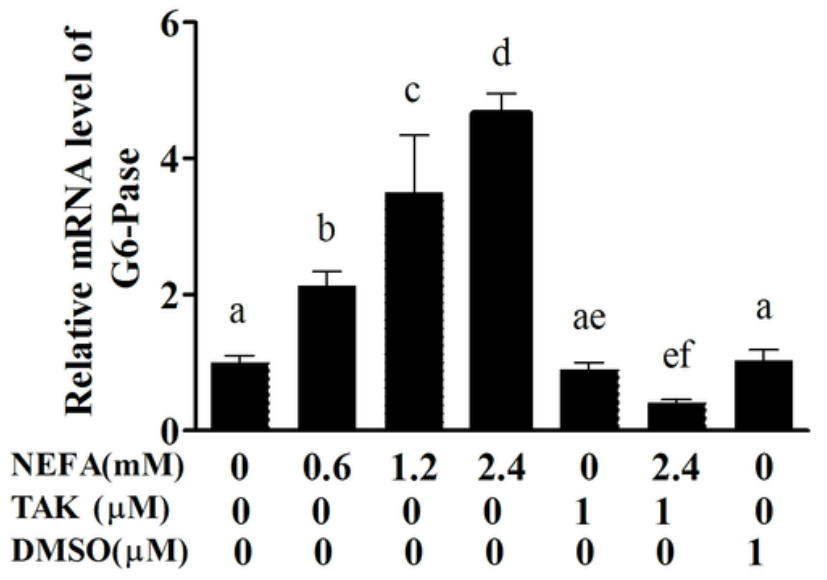

Figure 9

Effects of NEFAs and TAK-242 on the mRNA expression levels of PEPCK and G6-Pase. Values are the means \pm SEs $(n=6)$, and different letters indicate statistically significant differences; $p<0.05$.

A

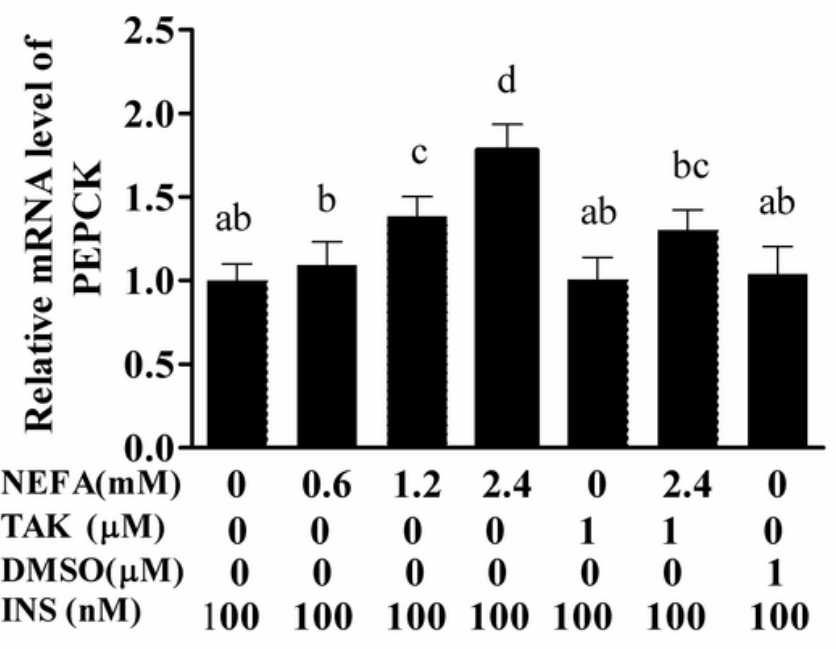

B

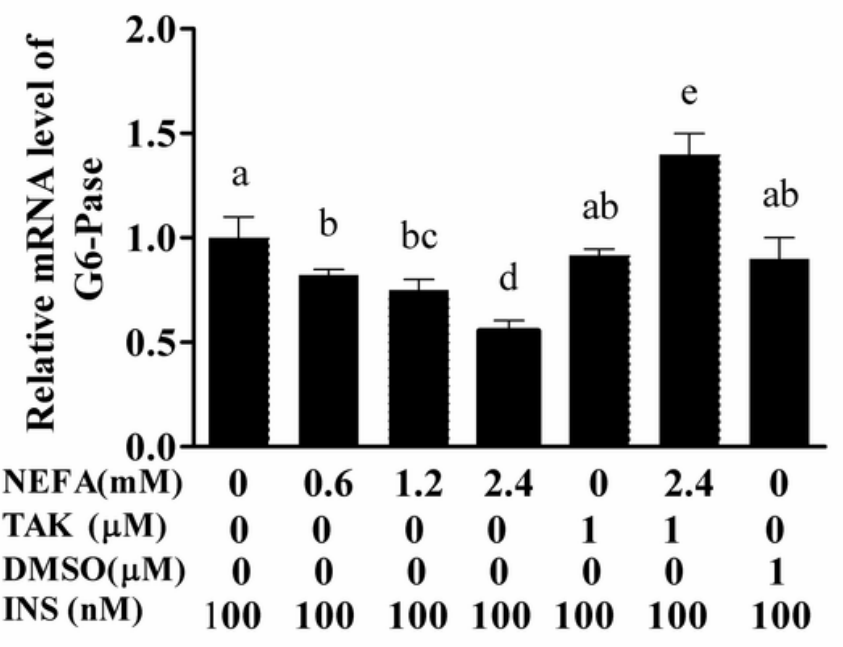

Figure 10

Effects of NEFAs, TAK-242 and INS on the mRNA expression levels of PEPCK and G6-Pase. Values are the means \pm SEs $(n=6)$, and different letters indicate statistically significant differences; $p<0.05$.

\section{Supplementary Files}

This is a list of supplementary files associated with this preprint. Click to download. 
- Supplementarymaterial.docx

Page 21/21 